\title{
Bienestar psicológico, espiritualidad en el trabajo y percepción subjetiva de la salud en personal académico y administrativo de la Universidad Nacional
}

\section{Psychological Well-Being, Spirituality at Work, and Self-Reported Health by Faculty and Staff from Universidad Nacional in Costa Rica}

\author{
Pedro Ureña Bonilla \\ Universidad Nacional \\ Escuela de Ciencias del Movimiento Humano y Calidad de Vida \\ Heredia, Costa Rica \\ purena 21@hotmail.com \\ Kristy Barrantes Brais² \\ Universidad Nacional \\ Escuela de Ciencias del Movimiento Humano y Calidad de Vida. \\ Heredia, Costa Rica \\ kristy.barrantes.brais@una.cr \\ Laura Solís Bastos ${ }^{3}$ \\ Universidad Nacional \\ Escuela de Ciencias del Movimiento Humano y Calidad de Vida \\ Heredia, Costa Rica \\ laura.solis.bastos@una.cr
}

Recibido 01 de abril de 2013 • Corregido 24 de setiembre de 2013 • Aceptado 01 de octubre de 2013

1 Doctor en Ciencias del Deporte por la Universidad de Deporte de Colonia, Alemania y Licenciado en Ciencias del Deporte. Universidad Nacional. Director de la Escuela de Ciencias del Movimiento Humano.

2 Licenciada en Psicología por la Universidad de Costa Rica. Máster en Salud Integral y Movimiento Humano por la Universidad Nacional. Académica, Escuela de Ciencias del Movimiento Humano y Calidad de Vida. Psicóloga, Departamento de Orientación y Psicología, Vicerrectoría de Vida Estudiantil Universidad Nacional.

3 Licenciada en Sociología por la Universidad Nacional de Costa Rica. Egresada de la Maestría en Estudios Latinoamericanos, por el Instituto de Estudios Latinoamericanos, Universidad Nacional de Costa Rica. Investigadora asociada de la Unidad de Investigación de la Escuela Ciencias del Movimiento Humano y Calidad de Vida. Asistente académica, Instituto de Estudios Sociales en Población (IDESPO), Universidad Nacional de Costa Rica. 
URL: http://www.una.ac.cr/educare

CORREO: educare@una.cr

Resumen. El propósito de este estudio fue describir y determinar la relación entre bienestar psicológico, espiritualidad en el trabajo y percepción de la salud en funcionarios de la Universidad Nacional. Se contó con la participación de 397 mujeres y 258 hombres $(n=655)$. La recolección de datos se hizo mediante la escala de bienestar psicológico, un cuestionario de autopercepción de la salud y la escala de espiritualidad en el trabajo. Se encontraron valores altos en propósito de vida $(6,08 \pm 1,08)$, auto-aceptación $(5,96 \pm 1,06)$, funcionamiento social $(2,47 \pm 0,37)$, significado del trabajo $(5,95 \pm 0,89)$ y vida interior $(5,81 \pm 1,09)$. Los valores promedio más bajos se registraron en las dimensiones relaciones interpersonales $(3,78 \pm 0,87)$, autonomía $(3,91 \pm 0,83)$ y dominio del entorno $(4,63 \pm 0,75)$. Asimismo, se registraron correlaciones significativas entre las dimensiones del bienestar psicológico, la percepción de la salud y la espiritualidad. En conclusión, es importante considerar los aspectos subjetivos en el bienestar de los trabajadores y las trabajadoras y señalar áreas donde es preciso intervenir para promover una universidad saludable.

Palabras claves. Calidad de vida, salud subjetiva, bienestar, espiritualidad.

Abstract. The purpose of this study was to determine and describe the relationship between psychological well-being, spirituality at work, and self-reported health by faculty and staff from Universidad Nacional in Costa Rica. Participants included 397 women and 258 men ( $n=655$ ). Data was collected using psychological well-being and spirituality at work scales and a self-reported health questionnaire. High values were found in the purpose in life (6.08 \pm 1.08$)$, self acceptance $(5.96 \pm 1.06)$, social functioning $(2.47 \pm 0.37)$, meaning of work $(5.95 \pm 0.89)$, and inner life $(5.81 \pm$ 1.09) categories. The lowest average values were reported in the following categories: interpersonal relationships ( $3.78 \pm 0.87)$, autonomy (3.91 \pm 0.83$)$, and environment mastery ( $4.63 \pm 0.75)$. Significant correlations were found between the psychological well-being, self-reported health, and spirituality dimensions. In conclusion, it is important to take into consideration the subjective aspects of personnel well-being and to determine which areas need intervention in order to promote a healthy university environment.

Keywords. Quality of life, subjective health, well-being, spirituality.

Desde el punto de vista psicológico, el concepto "universidad promotora de salud", atiende diferentes factores, entre los que ocupan lugar preponderante el bienestar psicológico, la espiritualidad y la sensación subjetiva de la salud (Arnold, Turner, Barling, Kelloway y Mckee, 2007; Romero, García-Mas y Brustand, 2009). Desde las propuestas de universidades promotoras de la salud, se ha definido que los aspectos psicosociales constituyen uno de los temas estratégicos por trabajar; se considera que entornos que permitan vivir y relacionarse saludablemente contribuyen a mejorar el bienestar social y emocional de toda la comunidad universitaria (Lange y Vio, 2006). En esta misma línea, se ha desarrollado el concepto "organizaciones saludables", entendidas como aquellas en las que se realizan prácticas saludables para estructurar y gestionar los procesos de trabajo, que influyen en el desarrollo de empleados saludables y comprometidos, así como en resultados organizacionales saludables (Salanova, 2008; Lorente y Vera, 2010). 
En este sentido, son varios los autores que reportan relaciones estrechas entre bienestar psicológico y percepción de la salud (Howell, Kern y Lyubomirsky, 2007; García-Viniegras, 2004; Labiano, 2004). De hecho, las personas más felices y satisfechas sufren menos molestias somáticas, tienen mejores apreciaciones personales, mejor dominio del entorno y poseen mejores habilidades sociales para vincularse con las demás y para crear comunidad (Eckersley, 2009). Así, la sensación de bienestar se asocia indefectiblemente con el funcionamiento psicológico positivo de las personas (Rodríguez-Carvajal, Díaz, Moreno-Jiménez, Blanco y van Dierendonck, 2010).

Íntimamente vinculada al concepto de bienestar, la espiritualidad en el trabajo es otro factor que incide poderosamente en la percepción subjetiva de la salud (Dein, 2005; Noor y Arif, 2011; Rodríguez, 2006; Sperry, Hoffman, Cox y Ervin, 2007). La espiritualidad tiene que ver con aspectos del lugar de trabajo que promueven sensaciones individuales de trascendencia y satisfacción que se proyectan al grupo y a la organización (Noor y Arif, 2011). Se trata de una experiencia trascendente de interconexiones, de completitud personal (Milliman, Czaplewski y Feguson, 2003; Noor y Arif, 2011). Es un factor que fortalece la vida interna de las personas $y$, consecuentemente, potencia la satisfacción laboral y la sensación subjetiva de salud. Altos niveles de espiritualidad les permiten a las personas una percepción atenuada de los estresores laborales y contribuyen al bienestar y a la salud (Rathi y Rastogi, 2007; Rodríguez, 2006). Congruente con estas afirmaciones, las personas con una buena espiritualidad tienen una autoestima positiva, un locus de control predominantemente interno, mecanismos efectivos para el manejo del estrés, altos niveles de tolerancia y mucha inteligencia emocional (Goldstein, 2007). Por lo tanto, promover la espiritualidad en el trabajo es fortalecer la sensación de bienestar (Arnold et al., 2007), la salud de los trabajadores (Sperry et al., 2007) y el rendimiento organizacional. En este sentido, autores como van der Walt (2007) señalan una relación estrecha y positiva entre satisfacción en el trabajo y espiritualidad. Todo parece indicar que altos grados de espiritualidad generan compromiso afectivo con la organización y satisfacción intrínseca en el trabajo (Moore y Casper, 2006).

De conformidad con lo planteado anteriormente, la valoración subjetiva de la salud está íntimamente relacionada con el bienestar psicológico (Albuquerque, 2004; Juárez, 2011). Por consiguiente, percibirse saludable equivale a sentir bienestar (Kahn y Juster, 2002), lo cual se asocia con una mayor capacidad adaptativa (Fierro, 2000) o mayor inteligencia emocional (Augusto-Landa y Montes-Berges, 2009; Bermúdez, Álvarez y Sánchez, 2003). No cabe duda que, en el marco de las organizaciones y las universidades saludables, la promoción de la espiritualidad y el bienestar son elementos consustanciales a la valoración subjetiva de la salud $y$, consecuentemente, determinantes de la calidad de vida de las personas.

Sobre la base de las argumentaciones precedentes, el propósito de este estudio fue analizar los niveles de bienestar psicológico, espiritualidad en el trabajo y percepción subjetiva de la salud en el personal académico y administrativo de la Universidad Nacional. 
URL: http://www.una.ac.cr/educare

CORREO: educare@una.cr

\section{Metodología}

\section{Población:}

En el estudio participaron 655 funcionarios y funcionarias de la universidad, 397 mujeres y 258 hombres. La edad promedio de los hombres fue de 39, 07 $\pm 9,54$ años y la de las mujeres fue de 39,99 $\pm 10,04$. El tiempo promedio de laborar para la Universidad fue de 9,8 \pm 8,0 años. En relación con el tipo de cargos, 373 personas se desempeñan en el área administrativa y 282 realizan tareas académicas. Del personal administrativo que participó en el estudio se registró un $48,6 \%$ de hombres y un $62,3 \%$ de mujeres. En cuanto al personal académico, el 51,5\% corresponde a hombres y el 37,7\% a mujeres. En lo concerniente a la condición laboral, el 43,3\% del personal encuestado es interino y el $56,7 \%$ es propietario. Asimismo, el $45,1 \%$ del personal interino corresponde a hombres y el $42,0 \%$ a mujeres. En el caso de quienes se encuentran en condición de propiedad, el $54,9 \%$ son hombres y el $58,0 \%$ son mujeres.

\section{Instrumentos:}

\section{Escala de bienestar psicológico (BP)}

Se utilizó la adaptación española realizada por Díaz et al. (2006). Es una escala de 29 ítems, a partir de la cual se registra información sobre las dimensiones: Autoaceptación $(\mathbf{a}=\mathbf{0 . 8 4})$, relaciones positivas con otros $(\alpha=0.78)$, autonomía $(\alpha=0.70)$, dominio del entorno $(\alpha=$ 0.82), propósito en la vida $(\alpha=0.70)$ y crecimiento personal $(\alpha=0.71)$. Utiliza una escala tipo likert con puntuaciones comprendidas entre $1=$ totalmente de acuerdo y $7=$ totalmente en desacuerdo.

\section{Cuestionario de salud general de Goldberg}

Se basó en la adaptación cubana realizada por García-Viniegras (2004), la cual consta de 12 ítems mediante los cuales se registra información sobre dos grandes factores: salud general y funcionamiento social. El instrumento emplea una escala likert que va de 0 a 3 en las categorías de respuesta. Para este instrumento se reportan índices de confiabilidad y validez superiores a 0.80 .

\section{Escala de espiritualidad}

Consiste en un instrumento autoaplicado que permite el registro de diferentes factores relacionados con la espiritualidad en el trabajo (Ashmos y Duchon, 2000). En este estudio se trabajó con los 3 factores más relevantes reportados por los autores indicados: condiciones de comunidad $(a=0.85)$, significado del trabajo $(a=0.85)$ y vida interna $(a=0.80)$. Se utilizó una escala tipo likert de 7 puntos donde $1=$ totalmente en desacuerdo y $7=$ totalmente de acuerdo. 
URL: http://www.una.ac.cr/educare CORREO: educare@una.cr

\section{Procedimiento:}

La ejecución de la encuesta se llevó a cabo durante los meses de setiembre, octubre y noviembre del 2011.

Los instrumentos fueron entregados en la mayor parte de las instancias universitarias (escuelas, decanatos, institutos). En algunos casos se solicitó un espacio para la ejecución del instrumento en reuniones, asambleas, concejos, entre otros, donde se explicaron los detalles de la investigación y se solicitó la colaboración respectiva; en otros casos, las encuestas fueron entregadas en las respectivas instancias universitarias, para que las personas seleccionadas para el estudio las contestaran. Los cuestionarios fueron recogidos de manera personal y otros fueron remitidos vía correo interno de la Universidad hasta la oficina de la Unidad de Investigación, ubicada en la Escuela Ciencias del Movimiento Humano y Calidad de Vida. La participación de todas las personas fue voluntaria y siempre se garantizó la absoluta discrecionalidad en el uso de la información.

\section{Análisis de la información:}

Los datos recolectados se registraron en una base de datos y fueron procesados mediante el Paquete estadístico de ciencias sociales (SPSS), versión 17.0 para Windows. Se calcularon valores descriptivos (media y desviación estándar), correlaciones bivariadas de Pearson, t-student para grupos independientes y análisis de varianza para contrastar categorías muestrales según sexo, edad, condición profesional, estabilidad laboral y experiencia laboral universitaria.

\section{Resultados}

Los valores promedio correspondientes al bienestar psicológico oscilaron entre 3,78 y 6,08 en escala de 1 a 7 valores (tabla 1). El valor más alto se registra para el factor "propósito en la vida", mientras que el factor "relaciones positivas" evidencia el menor valor promedio. En cuanto a la valoración subjetiva de la salud, el valor promedio superior corresponde al factor "funcionamiento social". En relación con la espiritualidad, el promedio superior se registra para el factor "significado de trabajo"; como contraste el promedio más bajo se registra en el factor "condiciones comunitarias".

La comparación entre hombres y mujeres muestra diferencias significativas en los factores: "propósito en la vida", "crecimiento personal", "salud general" y "condiciones comunitarias". En los dos primeros factores, las mujeres evidencian valores promedio significativamente superiores a los hombres. En el caso del factor "salud general", los hombres manifiestan valores promedio superiores a las mujeres. En cuanto a la valoración de las "condiciones comunitarias", las mujeres presentan un valor promedio superior al de los hombres. En el resto de los factores analizados no se encuentran diferencias significativas según el sexo de los encuestados. 
URL: http://www.una.ac.cr/educare

CORREO: educare@una.cr

Tabla 1

Valores promedio, desviaciones típicas y contraste según sexo para las dimensiones psicológicas

\begin{tabular}{|c|c|c|c|c|}
\hline Variable & General & Femenino & Masculino & Significancia \\
\hline \multicolumn{5}{|l|}{ Bienestar psicológico } \\
\hline Auto-aceptación & $5,96 \pm 1,06$ & $6,00 \pm 1,05$ & $5,89 \pm 1,07$ & 0,18 \\
\hline Relaciones positivas & $3,78 \pm 0,87$ & $3,74 \pm 0,85$ & $3,84 \pm 0,90$ & 0,15 \\
\hline Autonomía & $3,91 \pm 0,83$ & $3,91 \pm 0,81$ & $3,92 \pm 0,87$ & 0,89 \\
\hline Dominio del entorno & $4,63 \pm 0,75$ & $4,66 \pm 0,76$ & $4,59 \pm 0,75$ & 0,28 \\
\hline Propósito en la vida & $6,08 \pm 1,05$ & $6,20 \pm 1,02$ & $5,91 \pm 1,06$ & 0,00 \\
\hline Crecimiento personal & $5,26 \pm 0,75$ & $5,31 \pm 0,71$ & $5,19 \pm 0,80$ & 0,04 \\
\hline \multicolumn{5}{|l|}{ Percepción de la salud } \\
\hline Salud general & $2,11 \pm 0,49$ & $2,07 \pm 0,51$ & $2,16 \pm 0,46$ & 0,03 \\
\hline Funcionamiento social & $2,47 \pm 0,37$ & $2,46 \pm 0,36$ & $2,49 \pm 0,37$ & 0,28 \\
\hline \multicolumn{5}{|l|}{ Espiritualidad } \\
\hline Vida interna & $5,81 \pm 1,09$ & $5,94 \pm 0,87$ & $5,97 \pm 0,92$ & 0,73 \\
\hline Significado de trabajo & $5,95 \pm 0,89$ & $5,29 \pm 1,16$ & $5,31 \pm 1,10$ & 0,80 \\
\hline Condiciones comunitarias & $5,30 \pm 1,14$ & $6,01 \pm 0,89$ & $5,50 \pm 1,28$ & 0,00 \\
\hline
\end{tabular}

Nota: Elaboración propia.

La lectura general de la tabla 2, muestra relaciones importantes entre los factores del bienestar psicológico y los constructos de la percepción subjetiva de la salud y espiritualidad en el trabajo. Estas relaciones son más evidentes en cuanto a los factores "auto-aceptación", "propósito en la vida" y"crecimiento personal". Estos tres factores se relacionan de forma positiva y significativamente con la percepción subjetiva de la salud general, funcionamiento social, significado del trabajo, condiciones comunitarias y vida interna. En todos estos casos, cuanto mayor ha sido el puntaje registrado en esos factores del bienestar general, también mayores han sido los puntajes en factores propios del constructo "valoración subjetiva de la salud" y "espiritualidad en el trabajo". En lo particular, los factores "relaciones personales" y "autonomía" mostraron correlaciones significativas y negativas con los factores del constructo valoración subjetiva de la salud. En ambos casos, altos puntajes en esos factores se asociaron con bajos puntajes en valoración de la salud general y en funcionamiento social. El factor autonomía, 
URL: http://www.una.ac.cr/educare CORREO: educare@una.cr

también se relaciona de manera significativa y positiva con vida interna, aspecto central del constructo espiritualidad. Finalmente, el factor correspondiente a dominio del entorno únicamente se relacionó con el factor vida interna perteneciente a la espiritualidad en el trabajo.

Tabla 2

Correlaciones entre factores de bienestar psicológico, espiritualidad en el trabajo y percepción subjetiva de la salud

\begin{tabular}{|c|c|c|c|}
\hline \multicolumn{2}{|c|}{ Factores del bienestar psicológico } & \multirow[t]{2}{*}{ Pearson } & \multirow[t]{2}{*}{ Probabilidad } \\
\hline & Percepción de la salud & & \\
\hline & Salud general & $r=0,35$ & $p \leq 0,01$ \\
\hline & Funcionamiento social & $r=0,39$ & $p \leq 0,01$ \\
\hline \multirow[t]{5}{*}{ Autoaceptación } & Espiritualidad & & \\
\hline & Significado de trabajo & $r=0,35$ & $\mathrm{p} \leq 0,05$ \\
\hline & Condiciones comunitarias & $r=0,26$ & $p \leq 0,01$ \\
\hline & Vida interna & $r=0,35$ & $\mathrm{p} \leq 0,01$ \\
\hline & Percepción de la salud & & \\
\hline \multirow[t]{5}{*}{ Relaciones personales } & Salud general & $r=-0,16$ & $\mathrm{p} \leq 0,01$ \\
\hline & Funcionamiento social & $r=-0,22$ & $\mathrm{p} \leq 0,01$ \\
\hline & Percepción de la salud & & \\
\hline & Salud general & $r=-0,15$ & $p \leq 0,01$ \\
\hline & Funcionamiento social & $r=-0,19$ & $\mathrm{p} \leq 0,01$ \\
\hline \multirow[t]{7}{*}{ Autonomía } & Espiritualidad & & \\
\hline & Vida interna & $r=0,08$ & $\mathrm{p} \leq 0,05$ \\
\hline & Espiritualidad & & \\
\hline & Vida interna & $r=0,11$ & $p \leq 0,01$ \\
\hline & Percepción de la salud & & \\
\hline & Salud general & $r=0,30$ & $\mathrm{p} \leq 0,01$ \\
\hline & Funcionamiento social & $r=0,36$ & $\mathrm{p} \leq 0,01$ \\
\hline \multirow[t]{7}{*}{ Propósito en la vida } & Espiritualidad & & \\
\hline & Significado de trabajo & $r=0,32$ & $\mathrm{p} \leq 0,05$ \\
\hline & Condiciones comunitarias & $r=0,23$ & $\mathrm{p} \leq 0,05$ \\
\hline & Vida interna & $r=0,37$ & $\mathrm{p} \leq 0,01$ \\
\hline & Percepción de la salud & & \\
\hline & Salud general & $r=0,10$ & $\mathrm{p} \leq 0,01$ \\
\hline & Funcionamiento social & $r=0,11$ & $\mathrm{p} \leq 0,01$ \\
\hline \multirow[t]{4}{*}{ Crecimiento personal } & Espiritualidad & & \\
\hline & Significado del trabajo & $r=0,14$ & $p \leq 0,01$ \\
\hline & Condiciones comunitarias & $r=0,08$ & $\mathrm{p} \leq 0,01$ \\
\hline & Vida interna & $r=0,16$ & $\mathrm{p} \leq 0,01$ \\
\hline
\end{tabular}

Nota: Elaboración propia. 
URL: http://www.una.ac.cr/educare

CORREO: educare@una.cr

De acuerdo con la información contenida en la figura 1, existen correlaciones positivas y significativas entre los factores de los constructos valoración subjetiva de la salud y espiritualidad en el trabajo. De manera que altos puntajes en la valoración de la salud se asocian con altos puntajes en espiritualidad.

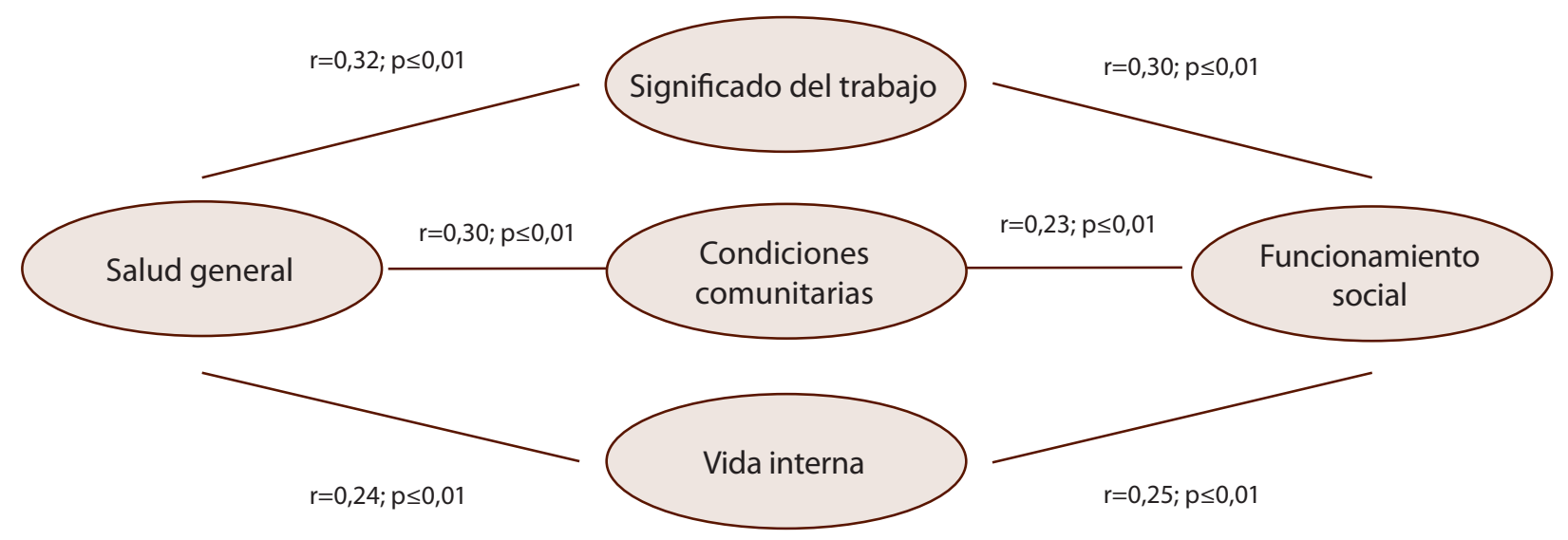

Figura 1. Correlaciones entre los constructos valoración subjetiva de la salud y espiritualidad en el trabajo.

Estudio propio (2012).

Nota: Elaboración propia

Asimismo, se encontraron diferencias significativas entre personal académico y administrativo en relación con el factor "significado del trabajo" (administrativo $=5,85$ $\pm 0,92$ / académico $=6,09 \pm 0,83 ; p<0,05$ ). En relación con los constructos espiritualidad en el trabajo y percepción subjetiva de la salud no se registraron diferencias entre personal académico y administrativo $(p>0,05)$. Tampoco el contraste según condición laboral (propietario / interino) y edad (22 - 33 años / 33,1 - 44 años / 44,1 - 79 años) evidenció diferencias estadísticamente importantes en relación con los factores de los constructos estudiados $(p>0,05)$. Con base en el criterio experiencia laboral ( $\leq 5$ años / 5,1 - 10 años / $\geq 10,1$ ) se identificaron datos significativos en cuanto al factor "condiciones comunitarias" del constructo espiritualidad en el trabajo. Las personas encuestadas con menos de 5 años de experiencia laboral en la Universidad Nacional, comparadas con las que tienen más de 5 años, mostraron valores promedio superiores en cuanto a la valoración que hacen de las condiciones generadoras de comunidad en el contexto universitario.

\section{Discusión}

En general, el personal universitario que participó en la encuesta mostró un perfil caracterizado por valores altos en propósito de vida, auto-aceptación, crecimiento personal, funcionamiento social, significado del trabajo, vida interna y condiciones comunitarias; todos 
ellos aspectos fundamentales para la calidad de vida de los trabajadores y las trabajadoras (Brannon y Feist, 2010; Marks et al., 2008; Straub, 2007; Vinaccia et al., 2005). Como áreas relativamente débiles se evidenciaron los factores: relaciones positivas, autonomía y dominio del entorno; aspectos que podrían afectar el soporte social, el locus de control interno y en general la percepción de la salud (Brannon y Feist, 2010; Marks et al, 2008).

En relación con el factor "propósito en la vida", se deduce la presencia de metas en los funcionarios encuestados, lo cual se vincula con el sentido de direccionalidad e intencionalidad. Congruente con estos resultados, Frankl (2011) es enfático al afirmar la importancia que tiene la sensación de sentido en la vida diaria. De manera que tener un propósito es un factor que potencia la salud y la calidad de vida (Klepp, Mastekaasa, Sorensen, Sandanger y Kleiner, 2007), a la vez le brinda perspectiva a la existencia del ser humano y le proporciona la energía necesaria para resolver las tareas de la cotidianidad (Antonovsky, 1996). Se ha encontrado que bajos puntajes en mediciones de bienestar psicológico, particularmente en las áreas de propósito en la vida y autoaceptación, podrían representar riesgo de depresión, por lo que se considera que puntuaciones altas podrían representar riesgo reducido de esta afección a la salud mental (Wood y Joseph, 2010).

Un segundo aspecto de importancia correspondió a los elementos relacionados con la autoaceptación y la sensación de autorrealización, lo que también se vincula con la idea de un buen funcionamiento en términos de capacidad adaptativa. La autorrealización representa, en términos de Maslow (1993), una de las necesidades fundamentales humanas y está vinculada a la sensación de logro en la vida (Klepp, Mastekaasa, Sorensen, Sandanger y Kleiner, 2007), lo que en aspectos retrospectivos se asocia con la existencia de propósito. En general, la percepción de una buena salud está unida a la sensación de autorrealización (Antonovsky, 1996; Maslow, 1993).

En relación con la autoaceptación, el tercer aspecto en orden de importancia correspondió al factor crecimiento personal, el cual se refiere a la capacidad adaptativa de la persona y se basa en el desarrollo continuo del potencial individual, es decir, en la realización personal (Maslow, 1993). Los hallazgos de este estudio también evidenciaron que las personas encuestadas no le dan tanta importancia a los aspectos del bienestar relacionados con las habilidades para establecer relaciones interpersonales. Esto podría considerarse como un elemento débil, por cuanto la capacidad de empatía es básica para la comprensión y buena convivencia entre las personas, aspectos que están vinculados a una buena salud (Bermejo, 2012). De hecho, mantener vínculos interpersonales brinda información que realimenta y fortalece la interacción entre las personas (Granovetter, 2000). En este contexto, toma importancia el concepto de redes de apoyo social, el que se basa en la capacidad de las personas para establecer relaciones interpersonales (Fernández, 2005). Es importante señalar que el apoyo social es una de las formas más efectivas de afrontamiento vinculadas al bienestar psicológico y a la sensación subjetiva de salud (Feldman et al., 2008; Gracia y Herrero, 2006; Kripper y Sapag, 2009; Mariscal, García, Fontaneda y González, 2010). 
Otro de los factores donde las personas participantes del estudio mostraron puntajes bajos correspondió al factor autonomía, lo que podría asociarse con un locus de control interno débil, por lo que podrían acusarse deficiencias en el proceso de regulación interna del comportamiento (Ajzen, 2002; Brannon y Feist, 2010). Esto podría significar que los encuestados y las encuestadas son susceptibles a la influencia y aprobación de terceras personas, condición que se reporta vinculada a limitaciones adaptativas que podrían atentar contra la salud (Marks et al., 2008). Valga señalar que la capacidad de autodeterminación está vinculada a la sensación de locus de control interno (Medellín, 2010; Molinero, Salguero y Márquez, 2011; Moreno y Martínez, 2006), por lo que su desarrollo debe ser estimulado. El locus de control interno potencia la responsabilidad, el compromiso y se asocia con una mejor calidad de vida (Medellín, 2010) y una mayor habilidad para crear y transformar las condiciones prevalentes, otorgándole a la persona una mayor adaptabilidad, característica fundamental para sentirse bien consigo misma y para crecer como persona. También se mostró como relativamente débil la capacidad para enfrentar las dificultades de la vida cotidiana y para tomar decisiones oportunas. En este sentido, es preciso señalar que el uso de mecanismos de afrontamiento adaptativos resulta fundamental para un mejor ajuste social, una mejor salud y calidad de vida (Straub, 2007).

Todos los valores encontrados en relación con la espiritualidad en el trabajo fueron altos, lo que refleja un grupo de personal académico y administrativo con una fuerte sensación de trascendencia y satisfacción. En relación con este constructo, fueron particularmente fuertes los sentimientos vinculados con el significado del trabajo y con la vida interna. El significado del trabajo resultó ser uno de los factores más importantes, lo que permite inferir que las personas encuestadas le encuentran sentido a su trabajo, lo que le da a la jornada laboral la connotación de auto-trascendente y la convierte en una actividad saludable (Gómez, Medina y Aguilar, 2011; Quinceno y Vinnaccia, 2009). En general, se considera que el trabajo en la Universidad Nacional tiene sentido y propósito, lo que alienta en el desempeño laboral. De acuerdo con Frankl (2011), las cosas crecen en importancia cuando se les asigna un sentido, no tanto de naturaleza física o psíquica, sino de orden espiritual.

El segundo aspecto en que se identifican fortalezas en las personas encuestadas es el optimismo y el valor de la autoconciencia en relación con los valores personales. El optimismo como variable psicológica ha sido identificado como uno de los factores determinantes de la salud de las personas (Bakker, Rodríguez-Muñoz y Derks, 2012; Londoño, 2009; Martínez, Reyes, García y González, 2006; Salanova y López-Zafra, 2011; Vera-Villarroel y Guerrero, 2003), particularmente porque genera esperanza, sentimiento que brinda perspectiva y favorece la autorrealización (Galván, 2008). Estudios científicos han identificado el optimismo como predictor significativo de resultados de salud física positivos (Rasmussen, Scheier y Greenhouse, 2009): las personas más optimistas (en comparación con las pesimistas) muestran menores riesgos de cardiopatía coronaria, mortalidad relacionada con cardiopatía coronaria, mortalidad relacionada con cáncer y mortalidad total (Tindle et al., 2009).

En general, las personas participantes del estudio coinciden en que desde su perspectiva hay elementos en el ambiente laboral que propician la sensación de comunidad. No cabe 
duda que sentirse en comunidad representa un factor potenciador de la salud, por cuanto las personas se sienten seguras, acompañadas y de alguna manera protegidas (Martínez, Amador, Moreno y Musitu, 2011; Maya, 2012).

El análisis de correlaciones mostró vínculos importantes entre bienestar psicológico, percepción subjetiva de la salud y espiritualidad. De manera particular, las personas que mostraron poseer actitudes positivas hacia sí mismos, que tienen metas en la vida, por las que luchan intencionalmente y sienten que están desarrollando su potencial, se caracterizan también por sentimientos de bienestar y por la sensación de competencia percibida, para enfrentar las dificultades de la vida cotidiana. En este sentido, los textos científicos son prolíferos al señalar la relación entre bienestar psicológico y espiritualidad (Casullo, 2006; Casullo y Fernández, 2006; Martínez et al., 2006; Valiente-Barroso y García-García, 2010). Más allá de lo subjetivo, se ha llegado incluso a establecer relaciones importantes entre el bienestar psicológico y la respuesta somática positiva de las personas (Molina-García, Castillo y Pablos, 2007). Se ha encontrado que niveles altos de bienestar psicológico se correlacionan con menores concentraciones de marcadores de inflamación (Friedman y Ryff, 2012; Morozink, Friedman, Coe y Ryff, 2010) y que, en general, las manifestaciones de bienestar emocional se asocian con una reducción en la mortalidad en diversas poblaciones (Chida y Steptoe, 2008).

En este estudio el sentimiento de auto-aceptación también se asoció con el hecho de que las personas le encuentran mayor sentido a lo que hacen $y$, por consiguiente, lo disfrutan mucho más. Como mecanismo de afrontamiento, auto aceptarse equivale a ser consciente de las fortalezas y debilidades propias, mientras que negarse a esa realidad puede relacionarse con estados emocionales negativos y dañinos para la salud (Jarne y García, 2008; Neff, Kirkpatrick y Rude, 2007). Por otro lado, las personas con una mayor autoaceptación son más optimistas (Jarne y García, 2008), tienen una mayor conciencia sobre sus valores personales y encuentran más elementos que propician la convivencia en comunidad (Touriñán, 2005; Touriñán, 2008).

La habilidad para establecer relaciones interpersonales, la capacidad de autorregulación y la habilidad para transformar el mundo de manera creativa según condiciones existentes no parecen ser importantes para que las personas le encuentren sentido al trabajo y perciban elementos en el medio que propicien la sensación de comunidad. Pareciera que el sentido trascendente que se le asigna al trabajo está inspirado en motivos intrínsecos (García-Alandete, Gallego-Pérez y Pérez-Delgado, 2009), por lo que el tema de las relaciones personales ocuparía un segundo plano. Por otro lado, el sentido de comunidad depende de cuánto se compartan sentimientos y metas tanto en el nivel micro o inmediato de la relación, como a nivel macro, es decir, institucional (Maya, 2012); lo que subraya la importancia de entender la misión institucional y de interiorizar los valores que implica el cumplimiento de estos.

La autonomía y el dominio del entorno se asocian de manera importante con el nivel de conciencia de las personas sobre los valores personales y las preocupaciones de orden espiritual. Claro está que la sensación de autodeterminación e independencia son fundamentales para que las personas se fortalezcan en sus valores y adquieran una perspectiva comprometida y 
responsable consigo mismas y con tareas que les corresponda realizar en cualquier contexto (Vélez, 2009). La presencia de sentimientos de bienestar, afectos positivos y la competencia percibida para enfrentarse a la vida cotidiana se asocian al hecho de disfrutar el trabajo y de encontrarle significado, con niveles altos de optimismo y con la percepción de elementos que propician la sensación de comunidad. En ese sentido, numerosos reportes han dejado clara evidencia del vínculo entre factores como el optimismo y el bienestar (Cuadra y Florenzano, 2003; Remor, Amorós y Carrobles, 2006; Vásquez, Hervás, Rahona y Gómez, 2009).

El análisis de contrastes a partir de las variables sexo, tipo de personal (académico / administrativo), condición laboral (interina / propiedad), edad y experiencia laboral solo en pocos casos mostró diferencias significativas. En relación con el sexo, las mujeres comparadas con los hombres mostraron un mayor propósito de vida y de crecimiento personal. Es decir, las féminas le encuentran más sentido a lo que hacen, lo que permite suponer un mayor nivel de motivación. Por otro lado, las mujeres también mostraron un mayor entusiasmo por el desarrollo del potencial personal, por auto realizarse. Detrás de esta tendencia podría valorarse el papel que juega el momento histórico, donde la mujer ha venido ganando espacio en todos los escenarios producto de ese empuje por crecer, por ese deseo de autorrealización (Guzmán y Rodríguez, 2008). En este mismo sentido, las mujeres, en contraste con los hombres, muestran percibir en el entorno una mayor cantidad de elementos que propician la vida en comunidad. El peso de la cultura parece jugar un rol importante como criterio explicativo de estas diferencias (Arroyo, 2003). Sin embargo, en cuanto a la prevalencia del sentimiento de bienestar general, los hombres comparados con las mujeres arrojan mejores resultados. Este elemento de la valoración subjetiva de la salud favorece a los hombres por sobre las mujeres. Quizá la mujer es más dada a las preocupaciones que los hombres, lo que refuerza la percepción de bienestar manifestada por estos (Extremera y Fernández, 2003). Según tipo de funcionario, los académicos comparados con los administrativos mostraron encontrarle más significado al trabajo. Esto podría asociarse con una mayor motivación y nivel de disfrute en él. Un elemento que podría explicar este comportamiento es la poca consciencia que aún existe sobre la complementariedad de lo académico y administrativo (Arias, 2009). Pareciera que el entendimiento holístico y sistémico del quehacer universitario es un tema que debe fortalecerse en esta institución. En relación con la experiencia laboral, los funcionarios universitarios con menos experiencia laboral, comparados con los de mayor experiencia, perciben más elementos que propician la sensación de comunidad, por lo que la permanencia en la institución y las experiencias asociadas podrían tender a deteriorar las condiciones que permiten construir comunidad.

Como conclusión general, queda claro que la construcción de una universidad u organización saludable requerirá de estrategias de intervención planificadas y sistemáticas, donde los factores psicosociales vinculados a la subjetividad de los trabajadores y las trabajadoras de la universidad ocupará un lugar preponderante. Siguiendo las recomendaciones de Lange y Vio (2006), se considera pertinente generar espacios para el desarrollo o fortalecimiento de habilidades personales y sociales, de relaciones interpersonales, así como 
de estilos de comunicación adecuados y resolución de conflictos. La promoción de igualdad de oportunidades, el establecimiento de mecanismos de participación democrática, y el desarrollo de procedimientos y políticas para la detección y atención de situaciones de acoso son algunas áreas en las que existen avances significativos que contribuirían a la creación y mantenimiento de ambientes promotores de bienestar y salud en la población universitaria.

Desde la perspectiva de una organización saludable, resultaría relevante la implementación de intervenciones dirigidas al personal, así como acciones desde la gestión organizacional con un enfoque positivo (Lorente y Vera, 2010; Salanova, 2008). Lo anterior implica tomar en cuenta el capital psicológico (fortalezas existentes) de la universidad y promover el aumento del bienestar a partir de intervenciones centradas en cogniciones (entrenamiento en toma de decisiones, generación y ensayo de pensamientos positivos), conductas (fortalecimiento de redes sociales, puesta en práctica de fortalezas, cuidado de la salud) y motivaciones (establecimiento de metas personales, estrategias de afrontamiento o desarrollo de la resiliencia, búsqueda de equilibrio entre retos y competencias).

Se considera que la incorporación de intervenciones en la cotidianidad laboral tendría que realizarse a partir de una comprensión de la forma en cómo se vivencia esta realidad. La sistematización de la experiencia del trabajador o trabajadora en contexto debería constituirse en una actividad recurrente que facilite la verbalización del pensamiento, emociones y afectos en las diferentes situaciones de la vida laboral universitaria. Apostarle a un enfoque fenoménico en la promoción de la salud y calidad de vida de los trabajadores universitarios tendría un valor agregado de gran cuantía. La inversión en el bienestar del personal universitario constituye una práctica saludable, que redundará en una organización que pueda aproximarse progresivamente a niveles de funcionamiento óptimo para beneficio de la comunidad universitaria y su entorno.

\section{Referencias}

Ajzen, I. (abril, 2002). Perceived Behavioral Control, Self-Efficacy, Locus of Control, and the Theory of Planned Behavior [La percepción de control del comportamiento, autoeficacia, locus de control, y la teoría de la conducta planeada]. Journal of Applied Social Psychology, 32(4), 665-683.

Albuquerque, A. S. (2004). Bem-estar subjetivo e sua relação com personalidade, coping, suporte social, satisfação conjugal e satisfação no trabalho [Bienestar subjetivo y su relación con personalidad, afrontamiento, soporte social, satisfacción conyugaly satisfacción en el trabajo] (Tesis doctoral), Instituto de Psicologia, Universidade de Brasilia.

Antonovsky, A. (1996). The Salutogenic Model as a Theory to Guide Health Promotion [El modelo salutogénico como una teoría a la guía de promoción de la salud]. Health Promotion International, 11(1), 11-18. Recuperado de http://heapro.oxfordjournals.org/ content/11/1/11.full.pdf+html 
Arias, C. E. (diciembre, 2009). Las "redes educativas regionales; un marco integral de gestión del conocimiento, para la reforma universitaria que contribuye a la transformación nacional". Revista de Ciencia y Tecnología, 5, 3-21. Recuperado de http://www.lamjol.info/index.php/ $\underline{\text { RCT/article/view/516/359 }}$

Arnold, K., Turner, N., Barling, J., Kelloway, K. y McKee, M. (Julio, 2007). Transformational Leadership and Psychological Well-Being: The Mediating Role of Meaningful Work [Liderazgo transformacional y bienestar psicológico: El papel mediador de un trabajo significativo]. Journal of Occupational Health Psychology, 12(3), 193-203.

Arroyo, A. (octubre, 2003). Cultura y género. Ponencia presentada en Seminario Internacional "Moneda, poder y sociedad: un enfoque multidisciplinario". Casa de las Humanidades de la Universidad Autónoma de México. Recuperado de http://fr.pekea-fr.org/sr-mx/Arroyo.pdf

Ashmos, D. y Duchon, D. (junio, 2000). Spirituality at Work: A Conceptualization and Measure [Espiritualidad en el trabajo: Una conceptualización y medición]. Journal of Management Inquiry, 9(2), 134-145. Recuperado de http://ejournal.narotama.ac.id/files/ ProQuest 54883215.pdf

Augusto-Landa, J. M. y Montes-Berges, B. (2009). Perceived Emotional Intelligence, Health and Somatic Symptomatology in Nursing Students [Inteligencia emocional percibida, salud y sintomatología somática en estudiantes de enfermería]. Individual Differences Research, 7(3), 197-211.

Bakker, A., Rodríguez-Muñoz, A. y Derks, D. (2012). La emergencia de la psicología de la salud ocupacional positiva. Psicothema, 24(1), 66-72. Recuperado de http://www.psicothema. com/pdf/3980.pdf

Bemerjo, J. C. (2012). Empatía terapéutica y compasión. Revista Chilena de Endocrinología y Diabetes, 5(2), 93-94. Recuperado de http://www.soched.cl/Revista\%20 Soched/2-2012/2-2012.pdf

Bermúdez, M. P., Álvarez, I. T. y Sánchez, A. (enero-junio, 2003). Análisis de la relación entre inteligencia emocional, estabilidad emocional y bienestar psicológico. Universitas Psychologica, 2(1), 27-32. Recuperado de http://sparta.javeriana.edu.co/psicologia/ publicaciones/actualizarrevista/archivos/V2N104analisis.pdf

Brannon, L. y Feist, J. (2010). Health Psychology. An introduction to Behavior and Health [Psicología de la salud. Introducción al comportamiento y la salud]. Wadsworth: Cengage Learning.

Casullo, M. M. (2006). El capital psíquico: Aportes de la psicología positiva. Psicodebate, 6, 59-72. Recuperado de http://www.palermo.edu/cienciassociales/publicaciones/pdf/ Psico6/6Psico\%2005.pdf 
Casullo, M. M. y Fernández, M. (2006). Las propuestas de la psicología positiva. ¿Universales psicológicos o particulares de una visión cultural? Anuario de investigaciones, 14, 261-268. Recuperado de http://www.scielo.org.ar/pdf/anuinv/v14/v14a25.pdf

Chida, Y. y Steptoe, A. (2008). Positive Psychological Well-Being and Mortality: A Quantitative Review of Prospective Observational Studies [Bienestar psicológico positivo y mortalidad: Revisión cuantitativa de estudios prospectivos observacionales]. Psychosomatic Medicine, 70, 741-756. doi: 10.1097/PSY.0b013e31818105ba

Cuadra, H. y Florenzano, R. (2003). El bienestar subjetivo: Hacia una psicología positiva. Revista de Psicología de la Universidad de Chile, 12(1), 83-86. Recuperado de http://www. revistapsicologia.uchile.cl/index.php/RDP/article/viewFile/17380/18144

Dein, S. (2005). Spiritualy, Psychiatry and Participation: A Cultural Analysis [Espiritualidad, psiquiatría y participación: Un análisis cultural]. Transcultural Psychiatry, 42(4), 526-544.

Díaz, D., Rodríguez-Carvajal, R., Blanco, A., Moreno-Jiménez, B., Gallardo, I., Valle, C. y van Dierendonck, D. (2006). Adaptación española de las escalas de bienestar psicológico de Ryff. Psicothema, 18(3), 572-577. Recuperado de http://www.psicothema.com/pdf/3255. pdf

Eckersley, R. (2009). Population Measures of Subjective Wellbeing: How Useful are they? [Medidas de población de bienestar subjetivo: ¿Qué utilidad tienen?]. Social Indicators Research, 94(1), 1-12. Recuperado de http://www.pratiquesciencessociales.net/exposes/ S3.\%20Population $\% 20$ Measures $\% 20$ of\%20Subjective $\% 20$ Wellbeing. $\% 20$ How $\% 20$ useful\%20are\%20they\%20(Eckersley\%202009).pdf

Extremera, N. y Fernández, P. (2003). La inteligencia emocional en el contexto educativo: Hallazgos científicos de sus efectos en el aula. Revista de Educación, 332, 97-116. Recuperado de http://www.mecd.gob.es/dctm/revista-de-educacion/articulosre332/ re3320611443.pdf?documentld=0901e72b81256ae3

Feldman, L., Goncalves, L., Chacón, G. C., Zaragoza, J., Bagés, N. y De Paulo, J. (setiembre-diciembre, 2008). Relaciones entre estrés académico, apoyo social, salud mental y rendimiento académico en estudiantes universitarios venezolanos. Universitas Psychologica, 7(3), 739-751. Recuperado de http://revistas.javeriana.edu.co/index.php/revPsycho/article/ view/406/282

Fernández, R. (2005). Redes sociales, apoyo y salud. Perifèria, 3, 1-16. Recuperado de http:// revistes.uab.cat/periferia/article/view/149/166

Fierro, A. (2000). Salud (comporta)mental: Un modelo conceptual. Revista de Psicología Generaly Aplicada, 53(1), 147-163. Recuperado e http://dialnet.unirioja.es/servlet/listaarticulos?tip oDeBusqueda $=$ VOLUMEN\&revistaDeBusqueda $=2008 \&$ claveDeBusqueda $=53$

Frankl, V. (2011). El hombre en busca de sentido. Barcelona: Grupo Planeta. 
Friedman, E. M. y Ryff, C. (2012). Living Well With Medical Comorbidities: A Biopsychosocial Perspective [Vivir bien con comorbilidades médicas: Una perspectiva biopsicosocial]. The Journals of Gerontology, Series B: Psychological Sciences and Social Sciences, 67(5), 535-544. doi: 10.1093/geronb/gbr152

Galván, L. (2008). Motivación: Estrategia de aprendizaje o autorrealización. Revista Digital de Investigación en Docencia Universitaria, 4(1), 1-17.

García-Alandete, J., Gallego-Pérez, J. F. y Pérez-Delgado, E. (mayo-agosto, 2009). Sentido de vida y desesperanza: Un estudio empírico. Universitas Psychologyca, 8(2), 447-454. Recuperado de http://revistas.javeriana.edu.co/index.php/revpsycho/article/view/218

García-Viniegras, C. (2004). Construcción y validación de una técnica para evaluar bienestar psicológico (Tesis doctoral). Instituto Superior de Ciencias Médicas de la Habana, Facultad Miguel Enríquez: Habana. Recuperado de http://promociondeeventos.sld.cu/psicosalud/ files/2008/03/resumen-tesis-carmen-regina-victoria.pdf

Goldstein, E. D. (2007). Sacred Moments: Implications on Well-Being and Stress [Momentos sagrados: Implicaciones en el bienestar y el estrés]. Journal of Clinical Psychology, 63(10), 1001-1019.

Gómez, M. I., Medina, B. y Aguilar, D. (2011). Sentido de vida y éxito. Uaricha Revista de Psicología, 8(17), 124-146. Recuperado de http://www.revistauaricha.org/Articulos/ uaricha 0817 124-146.pdf

Gracia, E. y Herrero, J. (2006). La comunidad como fuente de apoyo social: Evaluación e implicaciones en los ámbitos individual y comunitario. Revista Latinoamericana de Psicología, 38(2), 327-342. Recuperado de http://www.scielo.org.co/pdf/rlps/v38n2/ v38n2a07.pdf

Granovetter,M.S.(2000).Lafuerzadelosvínculos débiles.PolíticaySociedad,33,41-56. Recuperado de http://revistas.ucm.es/index.php/POSO/article/view/POSO0000130041A/24597

Guzmán, J. y Rodríguez, J. (2008). Comportamiento de las mujeres empresarias: Una visión global. Revista de Economía Mundial, 18, 381-392. Recuperado de http://www.redalyc.org/ articulo.oa?id=86601830

Howell, R., Kern, M. y Lyubomirsky, S. (2007). Health benefits: Meta-analytically Determining the Impact of Well-being on Objective Health Outcomes [Beneficios para la salud: determinación meta-analítica del impacto de bienestar sobre resultados objetivos de salud]. Health Psychology Reviews, 1(1), 83-136.

Jarne, A. y García, A. M. (febrero, 2008). Psicología positiva y salud. Comunicación presentada en Noveno Congreso Virtual de Psiquiatría. Interpsiquis. Palma de Mallorca: Intersalud.

Juárez, F. (2011). El concepto de salud: Una explicación sobre su unicidad, multiplicidad y los modelos de salud. International Journal of Psychological Research, 4(1), 70-79. Recuperado de http://mvint.usbmed.edu.co:8002/ojs/index.php/web/article/view/70/501 
URL: http://www.una.ac.cr/educare CORREO: educare@una.cr

Kahn, R. L. y Juster, F. T. (2002). Well-Being: Concepts and Measures [Bienestar: Conceptos y medidas]. Journal of Social Issues, 58(4), 627-644.

Klepp, O., Mastekaasa, A., Sorensen, T., Sandanger, I. y Kleiner, R. (2007). Structure Analysis of Antonovsky's Sense of Coherence from an Epidemiological Mental Health Survey with a Brief Nine-item Sense of Coherence Scale [Análisis estructural del sentido de coherencia de Antonovsky a partir de un estudio epidemiológico de salud mental con una escala breve de sentido de coherencia de nueve ítems]. International Journal of Methods in Psychiatric Research, 16(1), 11-22.

Kripper, C. y Sapag, J. (2009). Capital social y salud en América Latina y el Caribe: Una revisión sistemática. Revista Panamericana Salud Pública, 25(2), 162-170. Recuperado de http:// www.scielosp.org/scielo.php?script=sci arttext\&pid=S1020-49892009000200010\&lng=e n\&nrm=iso\&tlng=es

Labiano, M. (2004). Introducción a la psicología de la salud. En L. A. Oblitas (Aut.), Psicología de la salud y calidad de vida (pp. 3-19). México DF.: Thomson Editores.

Lange, I. y Vio, F. (2006). Guía para universidades saludables y otras instituciones de educación superior. Santiago: Organización Mundial de la Salud, Universidad de Chile, Pontificia Universidad Católica de Chile.

Londoño, C. (2009). Optimismo y salud positiva como predictores de la adaptación a la vida universitaria. Acta Colombiana de Psicología, 12(1), 95-107. Recuperado de http://www. redalyc.org/articulo.oa?id=79814903009

Lorente, L. y Vera, M. (julio-agosto, 2010). Las organizaciones saludables: “El engagement en el trabajo". Gestión Práctica de Riesgos Laborales, 73, 16-20.

Mariscal, M. A., García, S., Fontaneda, I. y González, O. (setiembre, 2010). La prevención del estrés laboral a través del apoyo social. XIV Congreso Ingeniería de Organización (pp. 64-651). Escuela Politécnica Superior. Universidad de Burgos. Recuperado de http://adingor. es/congresos/web/uploads/cio/cio2010/HEALTH AND OCCUPATIONAL SECURITY MANAGEMENT AND ERGONOMICS//644-651.pdf

Marks, D., Murray, M., Evans, B., Willing, C., Woodall, C. y Sykes, C. (2008). Psicología de la salud. Teoría, investigación y práctica. México: Manual Moderno.

Martínez, B., Amador, L.V., Moreno, D.y Musitu, G. (2011). Implicacióny participación comunitarias y ajuste psicosocial en adolescentes. Psicología y Salud, 21(2), 205-214. Recuperado de http://revistas.uv.mx/index.php/psicysalud/article/view/573/986 
Martínez, A., Reyes, G., García, A. y González, M.I. (2006). Optimismo/pesimismo disposicional y estrategias de afrontamiento del estrés. Psicothema, 18(1), 66-72. Recuperado de http:// www.psicothema.com/pdf/3177.pdf

Maslow, A. H. (1993). El hombre autorrealizado. Hacia una psicología del ser. Barcelona: Kairós.

Maya, I. (2012). Sentido de comunidad y potenciación comunitaria. Apuntes de psicología, 22(2), 187-211. Recuperado de http://personal.us.es/isidromj/php/wp-content/ uploads/2007/07/apuntes-sentido-de-comunidad.pdf

Medellín, E. W. (Julio-diciembre, 2010). Contrastación de dos modelos motivacionales de autodeterminación para predecir la deserción en universitarios. Acta Colombiana de Psicología, 13(2), 57-68. Recuperado de http://www.scielo.org.co/pdf/acp/v13n2/ v13n2a06.pdf

Milliman, J., Czaplewski, A. y Ferguson, J. (2003). Workplace Spirituality and Employee Work Attitudes: An Exploratory Empirical Assessment [Espiritualidad en el lugar de trabajo y actitudes laborales de los empleados: un estudio empírico exploratorio]. Journal of Organizational Change Management, 16(4), 226-447.

Molina-García, J. Castillo, I. y Pablos, C. (junio, 2007). Bienestar psicológico y práctica deportiva en universitarios. Motricidad. European Journal of Human Movement, 18, 79-91. Recuperado de http://www.redalyc.org/articulo.oa?id=274220374005

Molinero, O., Salguero, A. y Márquez, S. (julio, 2011). Autodeterminanción y adherencia al ejercicio: Estado de la cuestión. RICYDE. Revista Internacional de Ciencias del Deporte, 7(725), 287-304. Recuperado de http://www.redalyc.org/articulo.oa?id=71018867005

Moore, T. W. y Casper, W. (2006). An Examination of Proxy Measures of Workplace Spirituality: A Profile Model of Multidimensional Constructs [Un examen de las medidas de espiritualidad en el lugar de trabajo: Un modelo perfil de constructos multidimensionales]. Journal of Leadership \& Organizational Studies, 12(4), 109-118.

Moreno, J. A. y Martínez, A. (2006). Importancia de la teoría de la autodeterminación en la práctica físico-deportiva: Fundamentos e implicaciones prácticas. Cuadernos de psicología del deporte, 6(2), 39-54. Recuperado de http://revistas.um.es/cpd/article/view/113871/107881

Morozink, J. A., Friedman, E. M., Coe, C. L. y Ryff, C. D. (.2010). Socioeconomic and Psychosocial Predictors of Interleukin- 6 in the MIDUS National Sample [Predictores socioeconómicos y psicosociales de Interleucina-6 en la muestra nacional MIDUS]. Health Psychology, 29(6), 626-635. doi: 10.1037/a0021360

Neff, K., Kirkpatrick, K. y Rude, S. (2007). Self-compassion and Adaptive Psychological Functioning [Auto-compasión y el funcionamiento psicológico adaptativo]. Journal of Research in Personality, 41(1): 139-154. 
Noor, S. y Arif, S. (2011). Achievement Job Satisfaction VIA Workplace Spirituality: Pakistani Doctors in Focus [Satisfacción con logros laborales VIA espiritualidad en el lugar de trabajo: Enfoque en médicos paquistaníes]. European Journal of Social Sciences, 19(4), 507-515.

Quinceno, J. y Vinaccia, S. (2009). La salud en el marco de la psicología de la religión y la espiritualidad. Diversitas. Perspectivas en Psicología, 5(2), 321-336. Recuperado de http:// www.redalyc.org/articulo.oa?id=67916260008

Rasmussen, H. N., Scheier, M. F. y Greenhouse, J. B. (2009). Optimism and Physical Health: A Meta-analytic Review [Optimismo y salud física: Una revisión meta-analítica]. Annals of Behavioral Medicine, 37(3), 239-256. doi: 10.1007/s12160-009-9111-x

Rathi, N. y Rastogi, R. (enero, 2007). Meaning in Life and Psychological Well-being in Preadolescents and Adolescents [Sentido de la vida y bienestar psicológico en preadolescentes y adolescentes]. Journal of the Indian Academy of Applied Psychology, 33(1), 31-38.

Remor, E., Amorós, M. y Carrobles, J. A. (2006). El optimismo y la experiencia de ira en relación con el malestar físico. Anales de psicología, 22(1), 37-44. Recuperado de http://revistas. um.es/analesps/article/view/26231/25471

Rodríguez, M. I. (2006). Afrontamiento del cáncer y sentido de la vida: Un estudio empírico y clínico (Tesis doctoral). Universidad Autónoma de Madrid. Facultad de medicina. Recuperado de http://digitool-uam.greendata.es/exlibris/dtl/d3 1/apache media/ L2V4bGlicmlzL2R0bC9kM18xL2FwYWNoZV9tZWRpYS8xNDkx.pdf

Rodríguez-Carvajal, R., Díaz, D., Moreno-Jiménez, B., Blanco, A. y van Dierendonck, D. (2010). Vitalidad y recursos internos como componentes del constructo de bienestar psicológico. Psicothema, 22(1), 63-70. Recuperado de http://www.psicothema.com/pdf/3697.pdf

Romero, A. E., García-Mas, A. y Brustad, R. (2009). Estado del arte, y perspectiva actual del concepto de bienestar psicológico en psicología del deporte. Revista Latinoamericana de Psicología, 41(2), 335-347. Recuperado de http://www.redalyc.org/articulo.oa?id=80511496011

Salanova, M. (2008). Organizaciones saludables: Una aproximación desde la psicología positiva. En C. Vázquez y G. Hervás (Eds.), Psicología positiva aplicada (pp. 403-427). Madrid: Desclée de Brouwer. Recuperado de http://www.edesclee.com/pdfs/9788433022424.pdf

Salanova, M. y López-Zafra, E. (2011). Introducción: Psicología social y psicología positiva. Revista de Psicología Social, 26(3), 339-343. Recuperado de http://www.wont.uji.es/wont/ downloads/articulos/nacionales/2011SALANOVA21AN.pdf

Sperry, L., Hoffman, L., Cox, R. y Ervin, B. (2007). Spirituality in Achievement Physical and Psychological Health and Well-Being: Theory, Research and Low Cost Interventions [La espiritualidad en el logro de salud física, psicológica y bienestar: Teoría, investigación e intervenciones de bajo costo]. En L. L'Abate (Ed.), Low-Coast Approaches to Promote Physical and Mental Health Theory, Research and Practice [Enfoques de bajo costo para promover 
URL: http://www.una.ac.cr/educare

CORREO: educare@una.cr

teoría, investigación y práctica de la salud física y mental] (pp. 435-452). Recuperado de http://www.springerlink.com/content/g3l14113p475163k/fulltext.pdf

Straub, R. (2007). Health Psychology. A Biopsychosocial Approach [Psicología de la salud. Un enfoque biopsicosocial]. New York: Worth Publisher.

Tindle, H., Chang, Y-F., Kuller, L. Manson, J. E., Robinson, J. G., Rosal, M. C., .. Matthews, K. A. (2009). Optimism, Cynical Hostility, and Incident Coronary Heart Disease and Mortality in the Women's Health Initiative [Optimismo, hostilidad cínica, y cardiopatía y mortalidad coronaria incidente en la iniciativa de salud de la mujer]. Circulation, 120, 656-662. doi: 10.1161/CIRCULATIONAHA.108.827642

Touriñán, J. M. (2005). Experiencia axiológica y educación en valores. De la estimación personal del valor al carácter patrimonial de la elección de valores. Revista GalegoPortuguesa de Psicoloxía e Educación, 12(10), 9-43. Recuperado de http://ruc.udc.es/ dspace/bitstream/2183/7015/1/RGP 12-2.pdf

Touriñán, J. M. (2008). Educación en valores, educación intercultural y formación para la convivencia pacífica. España: Editorial Gesbiblo.

Valiente-Barroso, C. y García-García, E. (setiembre, 2010). La religiosidad como factor protector de salud y bienestar para un modelo multidisciplinario de atención psicogeriátrica. Psicogeriatría, 2(3), 153-165.

Van der Walt, F. (2007). The Relationship Between Spirituality and Job Satisfaction [Relaciones entre la espiritualidad y la satisfacción laboral] (Tesis doctoral inédita). University of Pretoria. Pretoria, Gauteng, South Africa.

Vásquez, C., Hervás, G., Rahona, J. J. y Gómez, D. (2009). Bienestar psicológico y salud: Aportaciones desde la psicología positiva. Anuario de Psicología Clínica y de la Salud APCS. 5, 15-28. Recuperado de http://institucional.us.es/apcs/doc/APCS 5 esp 15-28.pdf

Vélez, O. (2009). Líderes que trabajen por un mundo más equitativo, donde todos tengamos cabida: El respeto por la diferencia y la identidad. Poiésis, 17, 1-4. Recuperado de http:// www.funlam.edu.co/revistas/index.php/poiesis/article/viewFile/202/190

Vera-Villarroel, P. y Guerrero, A. (enero-junio, 2003). Diferencias en habilidades de resolución de problemas sociales en sujetos optimistas y pesimistas. Universitas Psycologica, 2(1), 21-26. Recuperado de http://sparta.javeriana.edu.co/psicologia/publicaciones/actualizarrevista/ archivos/V2N103diferencias.pdf 
Vinaccia, S., Quinceno, J. M., Fernández, H., Contreras, F., Bedoya, M., Tobón, S. y Zapata, M. (julio-diciembre, 2005). Calidad de vida, personalidad resistente y apoyo social percibido en pacientes con diagnóstico de cáncer pulmonar. Psicología y Salud, 15(2), 207-220. Recuperado de http://www.researchgate.net/publication/26472717 Calidad de vida personalidad resistente y apoyo social percibido en pacientes con diagnstico de cncer pulmonar

Wood, A. M. y Joseph, S. (2010). The Absence of Positive Psychological (Eudemonic) WellBeing as a Risk Factor for Depression: A Ten year Cohort Study [La ausencia de bienestar psicológico (eudaimónico) como un factor de riesgo para la depresión: Un estudio de cohorte de diez años]. Journal of Affective Disorders, 122(3), 213-217. doi:10.1016/j. jad.2009.06.032

\section{Cómo citar este artículo en APA:}

Ureña, P., Barrantes, K. y Solís, L. (enero-abril, 2014). Bienestar psicológico, espiritualidad en el trabajo y percepción subjetiva de la salud en personal académicos y administrativo de la Universidad Nacional. Revista Electrónica Educare, 18(1), 155-175. Recuperado de http://www.revistas.una.ac.cr/index.php/EDUCARE/issue/current

Nota: Para citar este artículo en otros sistemas puede consultar el hipervínculo "Como citar el artículo" en la barra derecha de nuestro sitio web:

http://www.revistas.una.ac.cr/index.php/EDUCARE/index 